\title{
Correction to: Preschool-Onset Major Depressive Disorder is Characterized by Electrocortical Deficits in Processing Pleasant Emotional Pictures
}

\author{
Diana J. Whalen ${ }^{1} \cdot$ Kirsten E. Gilbert $^{1} \cdot$ Danielle Kelly $^{1} \cdot$ Greg Hajcak $^{2} \cdot$ Emily S. Kappenman $^{3} \cdot$ Joan L. Luby $^{1}$. \\ Deanna M. Barch ${ }^{1,4,5,6}$
}

Published online: 2 March 2021

(c) Springer Science+Business Media, LLC, part of Springer Nature 2021

\section{Correction to: Journal of Abnormal Child Psychology (2020) 48:91-108. \\ https://doi.org/10.1007/s10802-019-00585-8}

The original version of this article unfortunately contained a mistake. The authors discovered an error in the processing of the ERP data included in originally published manuscript. All of the data has been reprocessed using average referencing. The primary findings in our original paper highlighted diagnostic group differences in ERPs from the 400-600 ms range but no group differences from $600-1000 \mathrm{~ms}$. We now report significant differences from $600-1000 \mathrm{~ms}$ and also a trend for significant group differences from 400-600 ms. One correlation between ERP responses to neutral pictures during the $400-600 \mathrm{~ms}$ window and BAS drives scores is no longer statistically significant, however this does not alter the conclusions made in the paper. A list of corrected results are provided below, as well as a revised Table 1 and Fig. 2. Please see the

The original article can be found online at https://doi.org/10.1007/ s10802-019-00585-8.

Diana J. Whalen

diana.whalen@wustl.edu

1 Department of Psychiatry, Washington University School of Medicine in St. Louis, 4444 Forest Park, Suite 2100, St. Louis, MO 63108, USA

2 Department of Psychology, Florida State University, Tallahassee, FL, USA

3 Department of Psychology, San Diego State University, San Diego, CA, USA

4 The Program in Neuroscience, Washington University in St. Louis, St. Louis, MO, USA

5 Department of Psychology, Washington University in St. Louis, St. Louis, MO, USA

6 Department of Radiology, Washington University in St. Louis, St. Louis, MO, USA online supplement for a completely edited results section of the manuscript. The original article has been corrected.

\section{Corrected Results}

- Trend effect of group on the pleasant-neutral difference score from $400-600 \mathrm{~ms}, F_{(1,147)}=3.14, p=0.08$, $\eta^{2}=0.02$.

- Group effect was found for the pleasant-neutral difference score from $600-1000 \mathrm{~ms}\left(F_{(1,147}=4.18, p=0.04\right.$, $\eta^{2}=0.03$ ).

- No significant sex differences were found for the pleasant-neutral differences score from $400-600 \mathrm{~ms}$ $\left(F_{(1,147}=0.09, p=0.77, \eta^{2}=0.001\right)$ or $600-1000 \mathrm{~ms}$ $\left(F_{(1,147)}=0.81, p=0.37, \eta^{2}=0.01\right)$.

- Trending effect of group on the residualized response to pleasant stimuli from $400-600 \mathrm{~ms}, F_{(1,147)}=2.98$, $p=0.09, \eta^{2}=0.02$.

- A group effect was found for the residualized response to pleasant $\left(F_{(1,147)}=3.96, p=0.04, \eta^{2}=0.03\right)$ stimuli from 600-1000 ms.

- No significant sex differences were found for the pleasantneutral differences score from $400-600 \mathrm{~ms}\left(F_{(1,147)}=0.01\right.$, $\left.p=0.92, \eta^{2}=0.00\right)$ or $600-1000 \mathrm{~ms}\left(F_{(1,147)}=1.08\right.$, $p=0.30, \eta^{2}=0.01$ ).

- When only correct trials were included, there was no main effect of diagnostic group on the pleasant-minus neutral difference score from $400-600 \mathrm{~ms}\left(F_{1,137}=0.26\right.$, $\left.p=0.61, \eta^{2}=0.002\right)$ or $600-1000 \mathrm{~ms}\left(F_{1,137}=0.67\right.$, $\left.p=0.41, \eta^{2}=0.01\right)$. There was no main effect of diagnostic group on the residualized response to pleasant stimuli from $400-600 \mathrm{~ms}\left(F_{1,137}=0.26, p=0.62, \eta^{2}=0.002\right)$ or $600-1000 \mathrm{~ms}\left(F_{1,137}=10.66, p=0.42, \eta^{2}=0.01\right)$.

- trend for BAS drive scores were positively correlated with mean amplitudes for Pleasant during the late segment $(r=0.19, p=0.06)$. 
Table 1 Raw and Residualized Event-Related Potential (ERP) Mean Scores during Pleasant and Neutral Picture Viewing

\begin{tabular}{|c|c|c|c|c|c|c|c|c|}
\hline \multirow{3}{*}{$\begin{array}{l}\text { ERP } \\
\text { Early: } 400-600 \mathrm{~ms}\end{array}$} & \multicolumn{4}{|c|}{ Healthy Control Children $(\mathrm{n}=58)$} & \multicolumn{4}{|c|}{ Depressed Children $(\mathrm{n}=90)$} \\
\hline & \multicolumn{4}{|c|}{ Amplitude $\mu \mathrm{V}$} & \multicolumn{4}{|c|}{ Amplitude $\mu \mathrm{V}$} \\
\hline & Mean & SD & Min & $\operatorname{Max}$ & Mean & SD & Min & $\operatorname{Max}$ \\
\hline Raw O1OzO2 Pleasant & 53.70 & 12.89 & 28.17 & 80.20 & 53.42 & 13.42 & 15.47 & 95.51 \\
\hline Residualized $\mathrm{O} 1 \mathrm{OzO} 2$ Pleasant & 1.05 & 6.55 & -12.51 & 24.48 & -0.33 & 6.17 & -20.51 & 13.09 \\
\hline Raw O1OzO2 Neutral & 49.65 & 12.63 & 25.19 & 93.66 & 50.88 & 13.10 & 16.33 & 91.28 \\
\hline Raw O1OzO2 Pleasant-Neutral Difference Score & 4.05 & 6.73 & -14.25 & 27.95 & 2.54 & 6.28 & -18.96 & 15.75 \\
\hline Late: $600-1000 \mathrm{~ms}$ & Mean & SD & Min & $\operatorname{Max}$ & Mean & SD & Min & Max \\
\hline Raw O1OzO2 Pleasant & 38.45 & 11.02 & 17.43 & 65.07 & 38.95 & 12.41 & 9.50 & 80.85 \\
\hline Residualized $\mathrm{O} 1 \mathrm{OzO} 2$ Pleasant & 0.44 & 5.46 & -11.74 & 24.53 & -0.68 & 6.23 & -23.95 & 14.62 \\
\hline Raw O1OzO2 Neutral & 34.43 & 11.02 & 15.56 & 75.68 & 36.16 & 11.56 & 11.70 & 74.16 \\
\hline Raw O1OzO2 Pleasant-Neutral Difference Score & 4.02 & 5.60 & -10.61 & 28.54 & 2.78 & 6.28 & -20.60 & 16.57 \\
\hline
\end{tabular}


Fig. 2 Scalp distribution of the difference between pleasant and neutral trials in the time-range of the LPP and ERPs of pleasant and neutral trials Healthy (Pleasant minus Neutral)
Healthy (Pleasant minus Neutral)
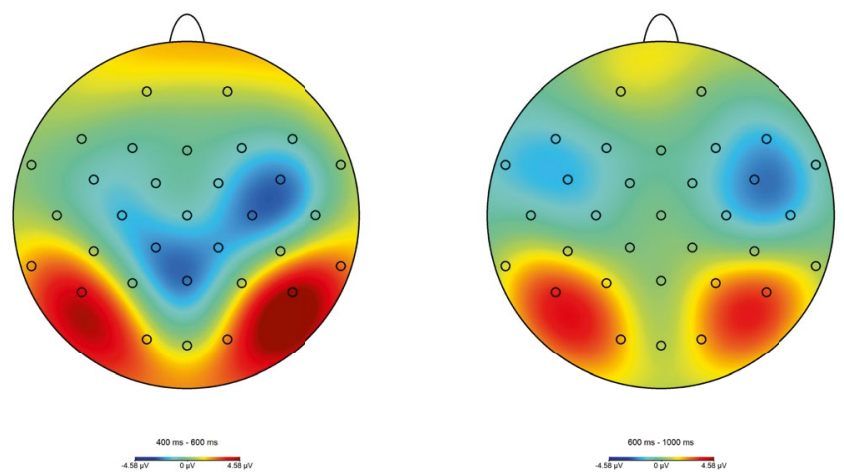

$-10$

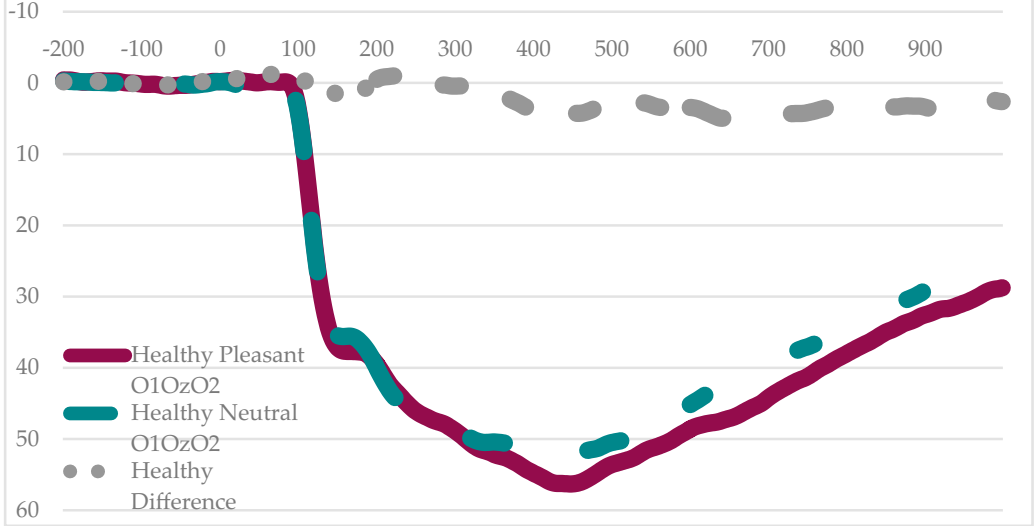

PO-MDD (Pleasant minus Neutral)
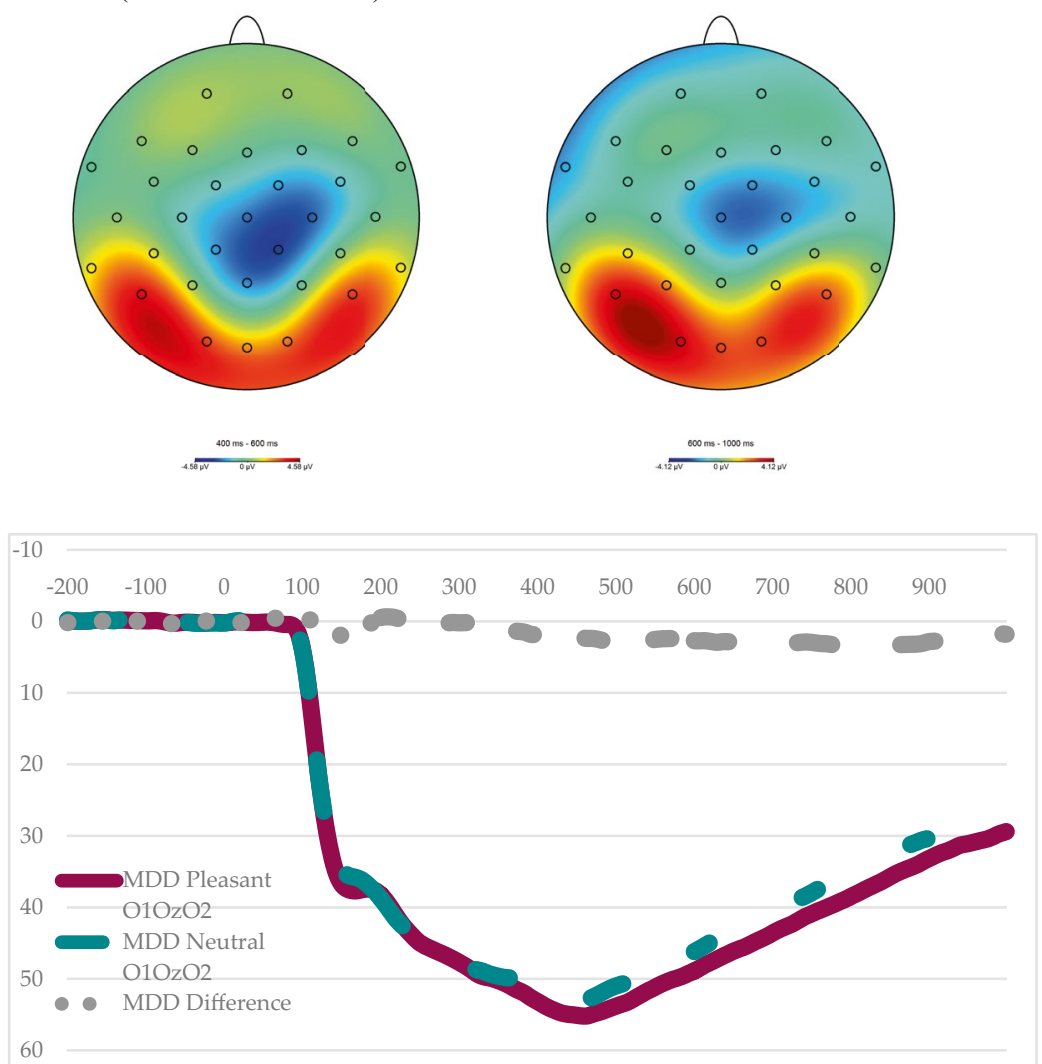
Supplementary Information The online version contains supplementary material available at https://doi.org/10.1007/s10802-021-00776-2.
Publisher's Note Springer Nature remains neutral with regard to jurisdictional claims in published maps and institutional affiliations. 